\title{
Micro-Cavity Fluidic Dye Laser
}

\section{Helbo, Bjarne; Kristensen, Anders; Menon, Aric Kumaran}

\section{Published in:}

IEEE The Sixteenth Annual International Conference on Micro Electro Mechanical Systems, 2003. MEMS-03 Kyoto.

Link to article, DOI:

10.1109/MEMSYS.2003.1189729

Publication date:

2003

Document Version

Publisher's PDF, also known as Version of record

Link back to DTU Orbit

Citation (APA):

Helbo, B., Kristensen, A., \& Menon, A. K. (2003). Micro-Cavity Fluidic Dye Laser. In IEEE The Sixteenth Annual International Conference on Micro Electro Mechanical Systems, 2003. MEMS-03 Kyoto. (pp. 235-238). IEEE. https://doi.org/10.1109/MEMSYS.2003.1189729

\section{General rights}

Copyright and moral rights for the publications made accessible in the public portal are retained by the authors and/or other copyright owners and it is a condition of accessing publications that users recognise and abide by the legal requirements associated with these rights.

- Users may download and print one copy of any publication from the public portal for the purpose of private study or research.

- You may not further distribute the material or use it for any profit-making activity or commercial gain

- You may freely distribute the URL identifying the publication in the public portal 


\title{
MICRO-CAVITY FLUIDIC DYE LASER
}

\author{
Bjarne Helbo $^{1}$, Anders Kristensen ${ }^{2}$, and Aric Menon ${ }^{3}$ \\ Mikroelektronik Centret (MIC) ${ }^{4}$, Technical University of Denmark (DTU) ${ }^{5}$ \\ Ørsteds Plads, Bldg. 345east, DK-2800 Kgs. Lyngby \\ Email: bh@mic.dtu.dk ${ }^{1}$ ak@mic.dtu.dk ${ }^{2}$ am@mic.dtu.dk ${ }^{3}$ \\ Web: http://www.mic.dtu.dk ${ }^{4} \quad$ http://www.dtu.dk/ ${ }^{5}$
}

\begin{abstract}
We have successfully designed, fabricated and characterized a micro-cavity fluidic dye laser with metallic mirrors, which can be integrated with polymer based lab-on-a-chip microsystems without further processing steps. A simple rate-equation model is used to predict the average pumping power threshold for lasing as function of cavity-mirror reflectance, laser dye concentration and cavity length. The laser device is characterized using the laser dye Rhodamine 6G dissolved in ethanol. Lasing is observed, and the influence of dye concentration is investigated.
\end{abstract}

\section{INTRODUCTION}

Liquid dye lasers [1] have been widely used as tunable, coherent light sources for spectroscopic analysis in the visible wavelength region - from app. $400 \mathrm{~nm}$ to $900 \mathrm{~nm}$. In this paper we report on the design and realization of a miniaturized liquid dye laser - a micro-cavity fluidic dye laser - which can be integrated with lab-on-a-chip microsystems.

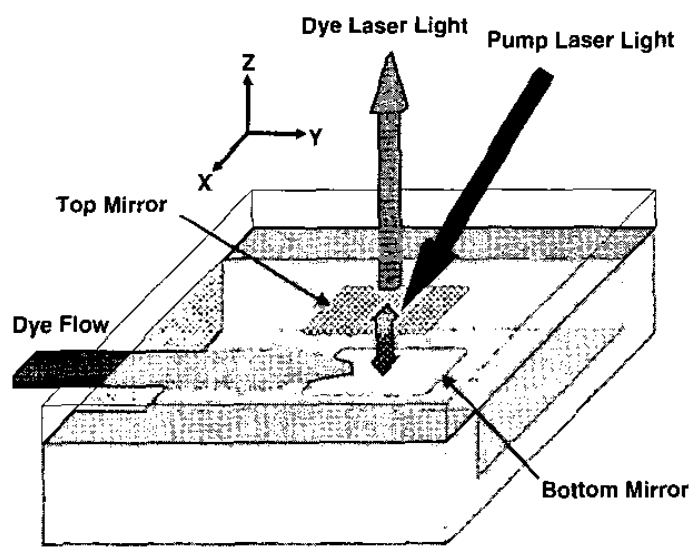

Figure 1: Vertically emitting micro-cavity fluidic dye laser. A liquid laser dye is pumped through a microfluidic channel, fitted with integrated mirrors forming the laser cavity. The dye is optically purmped by an external laser. Dye laser light is emitted through the glass lid.

The micro-fluidic dye laser consists of a micro-fluidic channel fitted with mirrors, forming the laser cavity. The laser dye is pumped through the micro-fluidic channel by means of an external pump, and the dye laser is optically pumped by an external laser. Dye laser output is emitted vertically from the device, as illustrated in figure 1 . In our design, the micro-fluidic channel structure is defined in a layer of photo-definable polymer (SU-8, [2]), sandwiched between two pyrex glass wafers. A Fabry-Perot laser cavity is formed by metallic mirrors, deposited on the top and bottom glass wafers by means of UV-lithography, e-beam evaporation and lift-off.

\section{DESIGN}

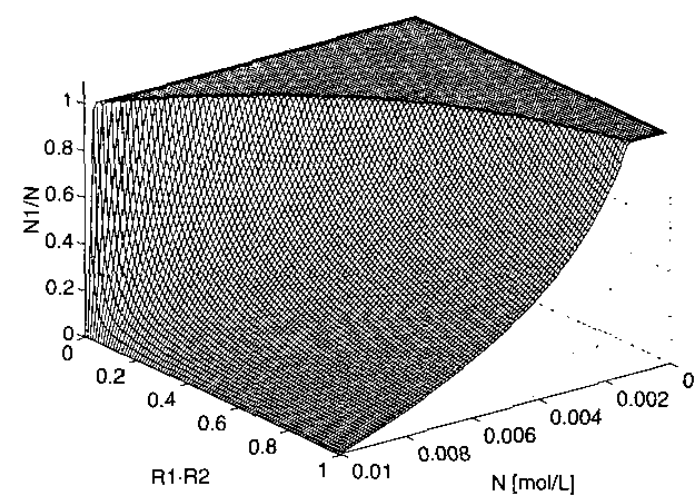

Figure 2: Surface plot of the critical inversion ratio $N_{1} / N$, which is the ratio between the concentration $N_{1}$ of the excited dye molecules, and $N$, the concentration of Rhodamine $6 \mathrm{G}$ dissolved in ethanol. The data are calculated for a cavity length, $L=10 \mu \mathrm{m} . N_{1} / N$ depends on the dye concentration $N$ and on the product $R_{1} \cdot R_{2}$ of the mirror reflectances. Lasing is only possible when $N_{1} / N \leq 1$, i.e. on the curved part of the surface. As seen on the plot, lasing is not possible for dye concentrations below $2 \cdot 10^{-3} \mathrm{~mol} / \mathrm{L}$.

The first design parameter, we consider, is the height, $H$ of the micro-fluidic channel. This is equal to the cavity length, $L_{\text {cavity }}$, which influences the optical modes and the round-trip gain in the laser cavity. Furthermore, it will also partly determine the optical stability of the resonator and the pressure drop across the micro-fluidic channel.

The round-trip gain can be calculated from rate equations [3]. The critical inversion ratio, $N_{1} / N$, is the ratio between the concentration of excited molecules, $N_{1}$, and the dye concentration, $N$, at lasing threshold. This 
is given by [4]:

$$
\frac{N_{1}}{N}=\frac{\sigma_{0}-\frac{1}{2 L_{\text {cavity } N}} \ln \left(R_{1} R_{2}\right)}{\sigma_{0}+\sigma_{e}+k_{\mathrm{ST}} \tau_{T}\left(\sigma_{0}-\sigma_{T}\right)}
$$

The rate equation and parameters for laser dyes are described in references [3] and [4]. $\sigma_{0}$ is the cross section for absorption of laser light by the ground state of the dye molecule, the singlet state. $\sigma_{e}$ is cross section for stimulated emission. $\sigma_{T}$ is the cross section for absorption of laser light due to the triplet state in the dye molecule. $L_{\text {cavity }}$ is the cavity length. $k_{\mathrm{ST}}$ is the intercrossing rate from the excited singlet state to the excited triplet state, and $\tau_{T}$ is the decay time from the excited triplet state to the singlet ground state. The critical inversion ratio at the lasing threshold, $N_{1} / N$, is calculated for a cavity length, $L_{\text {cavity }}=10 \mu \mathrm{m}$ and Rhodamine $6 \mathrm{G}$ dissolved in ethanol. The data are plotted as a so-called critical inversion surface in figure 2 . The parameters for Rhodamine $6 \mathrm{G}$ in ethanol are taken from $[1,3]$. As seen in figure 2 , lasing is possible in a $10 \mu \mathrm{m}$ long cavity for Rhodamine concentrations, $N$, above $2 \cdot 10^{-3} \mathrm{~mol} / \mathrm{L}$ with a reasonable laser cavity reflectance, $R_{1} \cdot R_{2}$. Cavity sizes much below $10 \mu \mathrm{m}$ will require higher dye concentrations, which will give problems with molecule to molecule stiction also called dimerization [1]. Dimer formation gives rise to extra optical losses in the laser dye.

Rhodamine $6 \mathrm{G}$ in ethanol fluoresces at wavelengths between $550 \mathrm{~nm}$ and $620 \mathrm{~nm}$. Using the value for ethanol, $n=1.36$, as a first estimate for the refractive index of the laser dye solution, and a cavity length of $L_{\text {cavity }}=10 \mu \mathrm{m}$, a total of 6 longitudinal cavity modes will overlap with the fluorescence spectrum.

The pressure drop $\Delta P$ in a micro-channel with a rectangular cross-section (assuming newtonian fluids) is given by [5]:

$$
\Delta P=8 \frac{\eta(W+H)^{2} L}{(W H)^{3}} \Phi=8 \frac{\eta(W+H)^{2} L}{(W H)^{2}} \bar{v}
$$

$W$ and $H$ are the width and the height, and $L$ is the length of the microfluidic channel. $\eta$ is the viscosity of the fluid. $\Phi$ is the flowrate and $\bar{v}$ is the flow speed. For a $10 \mu \mathrm{m}$ high channel the pressure will be fairly independent of the channel width, $W$, when this is larger than $1 \mathrm{~mm}$.

The cavity mirrors are designed with dimensions much larger than the cavity length in order to avoid problems with optical instability. A square top mirror with a side length of $2 \mathrm{~mm}$ is chosen which also provides sufficient coverage of a $1 \mathrm{~mm}$ wide channel. The bottom mirror covers the entire chip. Gold mirrors with chromium for adhesion was chosen due to ease of fabrication. The layer thickness of the mirrors were designed for specific reflectances using the method of Cory et al. [6] and data from Palik [7]. The bottom mirror was designed to have maximal reflectance $\left(R_{1}=0.83\right)$ and the top-mirror to be semi-transparent $\left(R_{2}=0.72\right)$ for both pump- and dye laser light, i.e. $R_{1} \cdot R_{2}=0.6$.
From the above considerations a cavity length of around $10 \mu \mathrm{m}$ and a cavity width of around $1 \mathrm{~mm}$ is reasonable. The flow channel length must be around $2.5 \mathrm{~cm}$ in order to accommodate the fluidic in- and outlet connections. These channel dimensions allows for flowrates up to around $200-300 \mu \mathrm{L} / \mathrm{min}$, if a pressure of $10 \mathrm{~atm}$ is considered as maximum.

\section{FABRICATION}

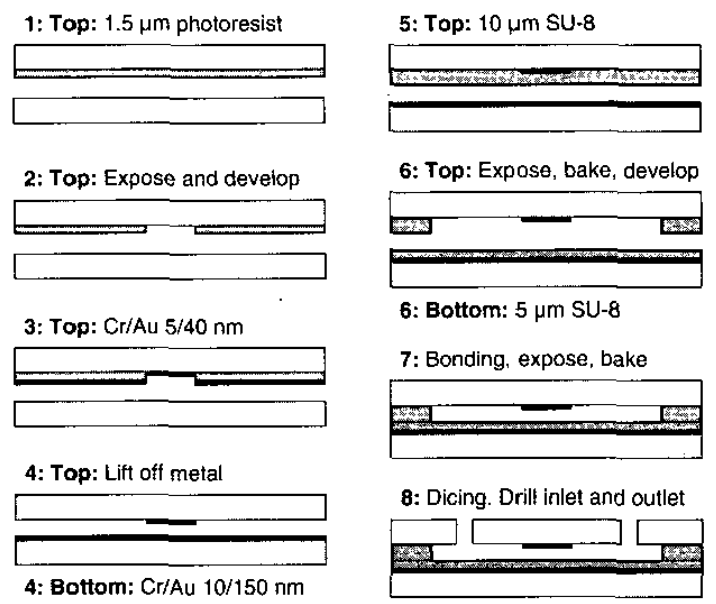

Figure 3: Fabrication process steps. $500 \mu \mathrm{m}$ thick Pyrex glass wafers are used as top and bottom substrates. 1-4: Metallic mirrors are deposited on the top- and bottom wafers by standard UV-lithography, electron-beam evaporation and lift-off. 5-6: The micro-flow channels are defined in SU-8 photoresist on the top wafer by spin-coating, softbaking baking, UV-exposure, post exposure baking and development. A $5 \mu \mathrm{m}$ thick SU-8 bonding layer is deposited on the bottom wafer by spin-coating and soft-baking. 7: The wafers are bonded by a manually applied pressure at $75^{\circ} \mathrm{C}$. The wafers are further sealed by a bake at $90^{\circ} \mathrm{C}$ for an hour and then cooled down before cross-linking of the SU-8 by an UV flood-exposure, followed by a post exposure bake. 8 : The chips are diced and inlet/outlet holes are drilled.

The process sequence is shown in figure 3. Pyrex glass wafers are used as top and bottom wafers, and in order to simplify the process we used $\mathrm{Cr} / \mathrm{Au}$ films for the mirrors. For the $\mathrm{Cr} / \mathrm{Au}$ films we have calculated the reflectance $R_{1}=83 \%$ (bottom), $R_{2}=72 \%$ (top) and the transmittance $T_{1}=0 \%, T_{2}=6 \%[6,7]$. Our process also allows for dielectric mirrors to be used. The semitransparent top mirror is defined in a lift off process. The microfluidic channel is defined in a $10 \mu \mathrm{m}$ thick SU-8 photoresist. The non transparent bottom mirror is deposited on the bottom wafer and a $5 \mu \mathrm{m}$ thick layer of $\mathrm{SU}-8$ is spun on as a bonding glue. Our low temperature SU- 8 bonding technique, which was partly adapted from Jackman et al. [8] and Shen et al. [9] requires no expensive bonding equipment and yet has a good hermetic quality. Both wafers in process step 6 are heated to $75^{\circ} \mathrm{C}$ and manually pressed together. The bonded wafers are baked at $90^{\circ} \mathrm{C}$ for an hour. This step improved the hermetic quality of the bonding. The wafers are again exposed to UV-light in order to cross-link the 
SU-8 on the bottom wafer and further cross-linked by a post exposure bake. The bonded wafers are diced before drilling inlet and outlet holes for the dye solution. A photo of a fabricated device is shown in figure 4 . The channel at the center of the chip is $1 \mathrm{~mm}$ wide and $10 \mu \mathrm{m}$ high.

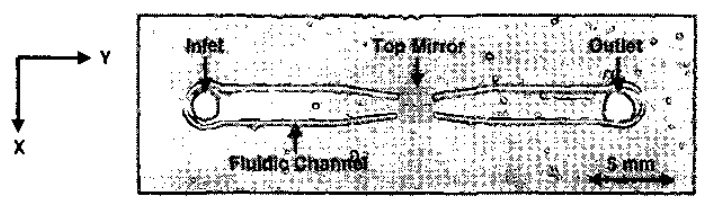

Figure 4: Photo of a glass/SU-8 chip with a micro-cavity fluidic dye laser, seen from above,

The chip is mounted in a plexiglass holder constructed with o-ring sealed fluid connections between the microfluidic channel and hoses to the external fluidic handling apparatus, as illustrated in figure 5.

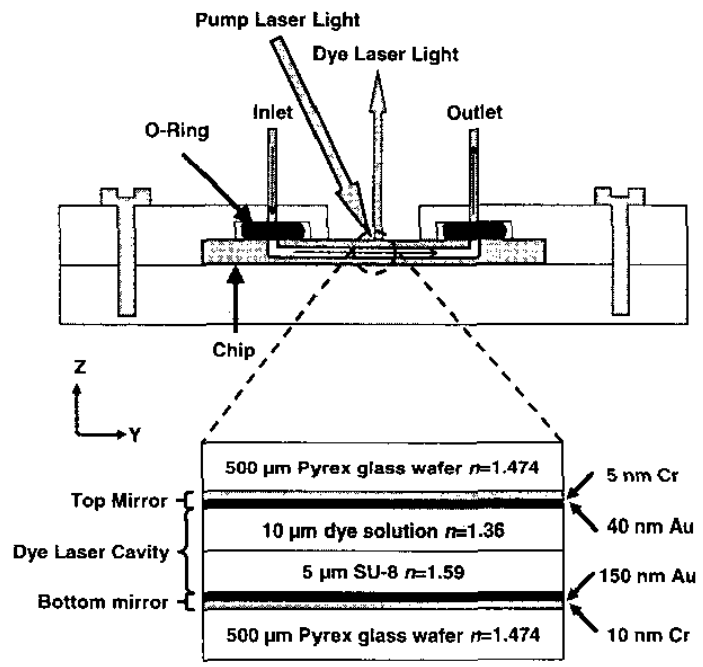

Figure 5: The micro-cavity fluidic dye laser chip is mounted in a plexiglass holder with o-ring sealed fluidic connections. The laser dye is pumped through the chip by means of an external syringe pump. The zoom-in shows a cross section of the laser cavity with the relevant layer thicknesses and refractive indices, $n$.

\section{RESULTS}

The laser was characterized using the laser dye Rhodamine $6 \mathrm{G}$ dissolved in ethanol as active medium. The micro-cavtiy fluidic dye laser was pumped optically by a pulsed, frequency doubled Nd:YAG laser (wavelength $532 \mathrm{~nm}$ ). The spot diameter of the pumping laser beam on the micro-cavity fluidic dye laser cavity was approximately $1.5 \mathrm{~mm}$. The dye laser output, emitted through the semi-transparent top mirror was collected with an optical fiber coupled to a spectrometer.

The influence of the dye concentration was investigated using six different dye concentrations ranging from $10^{-4}$ to $10^{-1} \mathrm{~mol} / \mathrm{L}$. The lowest concentration for which lasing was observed was $10^{-2} \mathrm{~mol} / \mathrm{L}$. Figure 6 shows a series of 5 optical spectra from the micro-cavity fluidic dye laser, using a dye solution of $5 \cdot 10^{-2} \mathrm{~mol} / \mathrm{L}$ Rhodamine $6 \mathrm{G}$ in ethanol and a flow rate of $10 \mu \mathrm{L} / \mathrm{min}$. The first peak, at $532 \mathrm{~nm}$ is due to scattered light from the pumping laser. The fluorescence from the Rhodamine $6 \mathrm{G}$ is seen in the wavelength range from app. $550 \mathrm{~nm}$ to $620 \mathrm{~nm}$. A strong peak around a wavelength of $578 \mathrm{~nm}$ emerges from the fluorescence, as the average pumping power is raised above $3 \mathrm{~mW}$. The insert compares three similar spectra, recorded with different laser dye concentrations. The strong peak is shifted towards longer wavelength for increasing dye concentration.

Figure 7 shows the corresponding peak average output power versus average pumping power for the three different dye concentration. For all three dye concentrations we observe a knee in the output versus input power trace. The knee, marked by a vertical arrow, is interpreted as the onset of lasing, and the corresponding values for the pumping power threshold for lasing $P_{\text {Ineas }}$ are tabulated in table 1.

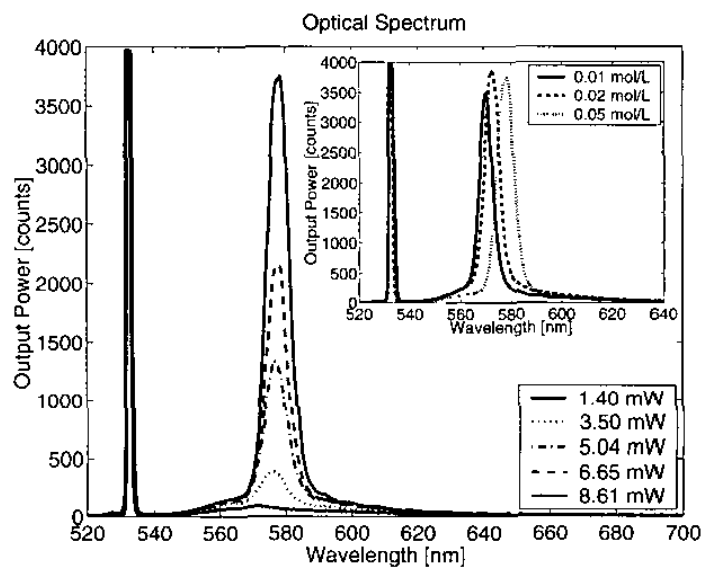

Figure 6: Measured spectra emitted from the micro-cavity fluidic dye laser, using a dye solution of $5 \cdot 10^{-2} \mathrm{~mol} / \mathrm{L}$ Rhodamine $6 \mathrm{G}$ in ethanol and a flow rate of $10 \mu \mathrm{L} / \mathrm{min}$. The spectra are recorded with different levels of optical pumping power (average power). The first peak at $532 \mathrm{~nm}$ is due to scattered pump laser light. A strong peak from the dye laser appears at around a wavelength of $578 \mathrm{~nm}$. The insert shows three dye laser spectra for three dye concentrations $10^{-2}, 2 \cdot 10^{-2}$, and $5 \cdot 10^{-2} \mathrm{~mol} / \mathrm{L}$. The lasing pcak wavelength is shifted towards longer wavelength for increasing dye concentration. The peak wavelength for the three spectra are: $570 \mathrm{~nm} .572 \mathrm{~nm}$, and $578 \mathrm{~nm}$, respectively.

\section{DISCUSSION}

The measured threshold average pumping powers, $P_{\text {meas }}$, are compared to the simple rate equation model presented previously. The pump laser is pulsed with a pulse length of $5 \mathrm{~ns}$ and a repetition rate of $10 \mathrm{~Hz}$. This is far from the stationary situation assumed in the model. However, the lifetime of the lasing photons in the cavity is in the range of 0.1 ps for our parameters 


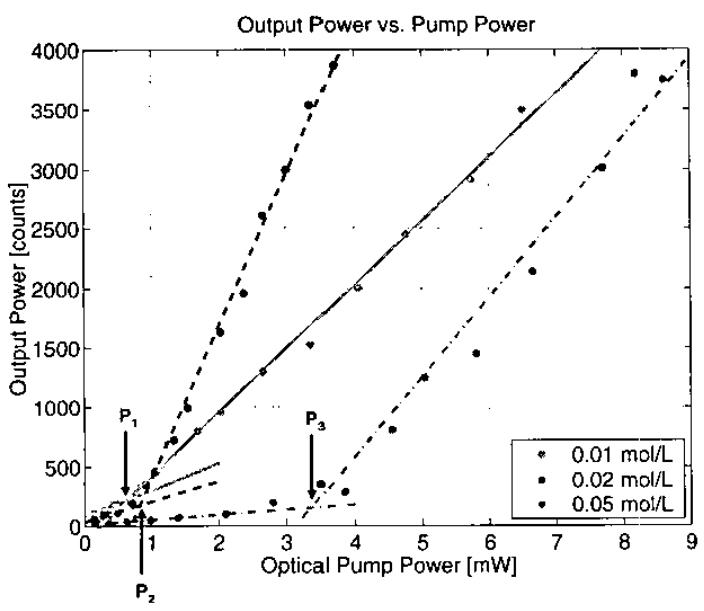

Figure 7: Measured lasing peak average output power versus average pumping power for the three dye concentrations $10^{-2}, 2 \cdot 10^{-2}$, and $5 \cdot 10^{-2} \mathrm{~mol} / \mathrm{L}$. Each trace exhibits a knee, which is interpreted as the onset of lasing. In order to extract a value for the threshold pumping power, $P_{i}$ (indicated on the plot), straight lines are fitted to the two parts of each trace. The extracted values are: $P_{1}=0.6 \mathrm{~mW}, P_{2}=$ $0.8 \mathrm{~mW}$, and $P_{3}=3.4 \mathrm{~mW}$

[3]. This is much shorter than the laser pulse length, and the model is used as an approximation. The calculated critical inversion population $N_{1}$, which can found from equation 1, can be used for calculating the corresponding threshold average pumping power $P_{\text {inv }}$. The following expression is obtained following Schäfer [1]:

$$
P_{\mathrm{inv}}=\frac{N_{1} h c L_{\mathrm{cavity}} A}{P_{\mathrm{conv}} T \lambda_{p} \tau_{0}}
$$

$h c / \lambda_{p}$ is the pumping photon energy. $L_{\text {cavity }}$ is cavity length and $A$ is the area of excitation on the dye laser cavity. $\tau_{0}$ is the fluorescent lifetime. $T$ is a constant, due to the reduced transmittance of the pumping light through the top mirror. For this case $T=0.07 . P_{\text {conv }}$ is a conversion factor from peak power to average power and is for our $\mathrm{Nd}$ :YAG laser approximately $20 \cdot 10^{6}$.

Table 1 shows the comparison between the measured average threshold pump power and the average threshold pump power predicted by the model for different dye concentrations. Due to the short pulsed pumping the

\begin{tabular}{|l|c|c|c|c|}
\hline$N[\mathrm{~mol} / \mathrm{L}]$ & $10^{-2}$ & $2 \cdot 10^{-2}$ & $5 \cdot 10^{-2}$ & $10^{-1}$ \\
\hline$P_{\text {meas }}[\mathrm{mW}]$ & 0.6 & 0.8 & 3.4 & 8.6 \\
\hline$P_{\text {model }}[\mathrm{mW}]$ & 0.96 & 0.96 & 0.97 & 0.98 \\
\hline
\end{tabular}

Table 1: Comparison betwcen measured average threshold pump power and the average threshold pump power predicted by the model for the critical inversion ratio for different concentrations. There is an agreement at lower concentrations but at higher concentrations the measured threshold exceeds the predicted values. This could be an indication of dimerization of the dye molecules due to the high concentrations. The model does not take dimerization into account.

triplet state can ignored, which has been done for the calculated values in table 1.

There is a good agreement between the measured values and the and model for the lower concentrations. However, at higher concentrations there is a large deviation. The measured values at high concentration are much higher than values predicted by the model. This can be an indication of dimerization due to the high dye concentrations. Dimerization is not accounted for in the model.

In conclusion, we have demonstrated a microcavity fluidic dye laser, fabricated by standard microfabrication techniques. The laser can easily be integrated with lab-on-a-chip microsystems. A simple rate equation model was used in the design considerations. The model shows, that lasing can be achieved in a $10 \mu \mathrm{m}$ long cavity at moderate concentrations of the laser dye Rhodamine $6 \mathrm{G}$ in ethanol. The model also predicts, that lasing is obtainable with low cavity mirror reflectance and for reasonable dye concentrations.

In our design, the laser dye is continuously pumped through the laser cavity. This allows for $\mathrm{cw}$ laser operation and a long lifetime for the system. Other groups have previously demonstrated micro-cavity dye lasers $[10,11]$, but to our knowledge, our design is the first one using standard micro-fabrication techniques for fluidic dye lasers.

\section{ACKNOWLEDGEMENTS}

This work was supported by the Danish Technical Research Council, STVF (grant number 26-02-0064), and by the H.C. Ørsteds Foundation.

\section{REFERENCES}

[1] F. P. Schäfer, Dye Lasers, Berlin: Springer-Verlag, 1990

[2] XP SU-8 photoresist and PGMEA developer from MicroChem Corp.

[3] J. Barroso, A. Costela, I. García-Moreno, and R. Sastre, Chemical Physics, 238, 257-272, 1999

[4] O. G. Peterson, J. P. Webb, W. C. McColgin, and J. H. Eberly, Journal of Applied Physics, 42, 12, 1917-1928, 1971

[5] A. E. Hatch, A. E. Kampholz, G. Holman, P. Yager, and K. F. Böhringer, Microelectromechanical System, 10, 2, 215-221, 2001

[6] H. Cory, S. Shiran, and M. Heilper, IEEE Transactions on Electromagnetic Compatibility, 35, 451-456, 1993

[7] E. D. Palik, Handbook of Optical Constants of Solids, New York: Academic Press, 1998

[8] R. J. Jackman, T. M. Floyd, R. Ghodssi, M. A. Schmidt, and K. F. Jensen, Journal of Micromechanics and Microengineering, 11 $263-269,2001$

[9] S. C. Shen, C. T. Pan, and H. P. Chou, Proceedings of SPIE, 4407, 185-192, 2001

[10] F. De Martini, G. Innocenti, G. R. Jacobovitz, and P. Mataloni, Physical Review Letters, 59, 2955-2958, 1987

[1]] H. Yokoyama, M. Suzuki, and Y. Nambu, Applied Physical Letters, 58, 2598-2600, 199] 